\title{
Hydrological Modeling of the Manglaralto River for the Generation of Flood Maps (Santa Elena-Ecuador)
}

\author{
F. Javier Montalván Toala, PhD ${ }^{1,2}$, M. Michelle Catuto Quinde, Ing ${ }^{1,2}$, and Gilda Rubira Gómez, MSc. ${ }^{2}$ \\ ${ }^{1}$ Universidad Estatal Península de Santa Elena, UPSE, Centro de Investigación e Innovación de Geociencias CIGEO, Av. \\ Principal Santa-La Libertad, Ecuador. fmontalvan@upse.edu.ec, mcatutoquinde@upse.edu.ec \\ ${ }^{2}$ Universidad Estatal Península de Santa Elena, UPSE, Facultad de Ciencias de la Ingeniería, Av. Principal Santa \\ Elena-La Libertad, Ecuador. grubira@upse.edu.ec
}

\begin{abstract}
The purpose of carrying out the hydrological modeling of the Manglaralto river is to locate the sites with the greatest risk due to flooding only due to rainfall in the basin, providing governmental and non-governmental authorities with support material that serves to analyze risks with basic technology for planning, and the analysis of flood damage reduction. The models were made for three types of scenarios, scenario 1 (natural situation), scenario 2 (current situation) and scenario 3 (future situation) in which the value of the curve number for each subbasin and the intervention of existing civil works influence and future. The calculated flood hydrographs were made in the HEC HMS program, necessary input data in the HEC RAS program for flow simulation for 24 hours, these are mathematical models based on the SCS soil conservation service methodology carried out by the Institute of United States Army used to prevent and mitigate impacts of natural events presented on maps that show overflow margins in the three situations cited with their respective flood maps zoned by the types of threat: high, medium and low.
\end{abstract}

Keywords: Mathematical models, maps, floods, river basins, hydrograms, rainfall.

Digital Object Identifier (DOI):

http://dx.doi.org/10.18687/LACCEI2020.1.1.213

ISBN: 978-958-52071-4-1 ISSN: 2414-6390

$18^{\text {th }}$ LACCEI International Multi-Conference for Engineering, Education, and Technology: "Engineering, Integration, and Alliances for a Sustainable Development" "Hemispheric Cooperation for Competitiveness and Prosperity on a Knowledge-Based Economy", 27-31 July 2020, Virtual Edition. 


\title{
Modelación Hidrológica del Río Manglaralto para la Generación de Mapas de Inundación (Santa Elena-Ecuador)
}

\author{
F. Javier Montalván Toala, PhD ${ }^{1,2}$, M. Michelle Catuto Quinde, Ing ${ }^{1,2}$, and Gilda Rubira Gómez, MSc. ${ }^{2}$ \\ ${ }^{1}$ Universidad Estatal Península de Santa Elena, UPSE, Centro de Investigación e Innovación de Geociencias CIGEO, Av. \\ Principal Santa-La Libertad, Ecuador. fmontalvan@upse.edu.ec, mcatutoquinde@upse.edu.ec \\ ${ }^{2}$ Universidad Estatal Península de Santa Elena, UPSE, Facultad de Ciencias de la Ingeniería, Av. Principal Santa \\ Elena-La Libertad, Ecuador. grubira@upse.edu.ec
}

\begin{abstract}
Resumen-El propósito de realizar la modelación hidrológica del río Manglaralto es ubicar los sitios con mayor riesgo por inundación únicamente por precipitaciones en la cuenca, proporcionando a las autoridades gubernamentales y no gubernamentales un material de apoyo que sirve para el análisis de riesgos con tecnología de base para la planificación, y el análisis de la reducción de daños por inundaciones. Los modelos se realizaron para tres tipos de escenarios, escenario 1(situación natural), escenario 2 (situación actual) y escenario 3 (situación futura) en los cuales influye el valor de número de curva para cada subcuenca y la intervención de obras civiles existentes $y$ futuras. Los hidrogramas de crecida calculados se realizaron en el programa HEC HMS datos de ingreso necesarios en el programa HEC RAS para la simulación de flujo durante 24 horas, Los hidrogramas de crecida calculados se realizaron en el programa HEC HMS datos de ingreso necesarios en el programa HEC RAS para la simulación de flujo durante 24 horas, estos son modelos matemáticos basados en la metodología de servicio de conservación de suelo SCS realizados por el Instituto del Ejercito de los Estados Unidos utilizados para prevenir y mitigar impactos de sucesos naturales presentados en mapas que visualizan márgenes de desbordamiento en las tres situaciones citadas con sus respectivos mapas de inundación zonificados por los tipos de amenaza: alta, media y baja.
\end{abstract}

Palabras claves: Modelos matemáticos, mapas, inundaciones, cuencas hidrográficas, hidrogramas, precipitaciones.

\section{INTRODUCCIÓN}

Ecuador es un país con diversidad climática, por ende, las precipitaciones son el elemento más importante para estudios hidrológicos e hidráulicos. La prevención y mitigación de impactos asociados con el uso de suelo son relevantes para el análisis y posterior toma de decisiones relacionados a los asentamientos y al crecimiento poblacional que aumenta cada año y esto lleva a controles respecto a su uso para prevención de inundaciones.

En Ecuador los recursos hídricos explotados alcanzan los $9700 \mathrm{hm}^{3}$, de los que $7954 \mathrm{hm}^{3}$ se usan para regadío, mientras que $1212.5 \mathrm{hm}^{3}$ para el uso doméstico y $533.5 \mathrm{hm}^{3}$ para la industria [1].

La cuenca del río Manglaralto drena hacía el océano Pacífico, siendo un significativo recurso hídrico ubicado al norte de la provincia de Santa Elena, habitualmente la población de este sector y sus proyecciones futuras de construcción y diseño aumentan con el tiempo, por lo que los habitantes del sector cubren sus necesidades exclusivamente con agua del subsuelo, perforando pozos en las terrazas aluviales de la cuenca.

Digital Object Identifier (DOI):

http://dx.doi.org/10.18687/LACCEI2020.1.1.213

ISBN: 978-958-52071-4-1 ISSN: 2414-6390
En la zona de estudio entre el periodo 2000-2015 la precipitación y la ETP corresponden a $389 \mathrm{~mm}$ y $1250 \mathrm{~mm}$ respectivamente, lo cual indica que existe un déficit hídrico en la cuenca del río Manglaralto, lo que es acorde a un clima semi-árido [2].

Los recursos hídricos superficiales de la cuenca no son aprovechados para ninguna actividad socioeconómica por parte de los habitantes del sector, ni actividad industrial. Este recurso superficial en los últimos cinco años ha sido aprovechado exclusivamente para la recarga artificial del acuífero Manglaralto, dado que los habitantes dependen del agua subterránea [3], [4], [5], [6], [7].

Los fenómenos físicos, en este caso las precipitaciones ocupan un lugar prioritario en la toma de decisiones que se han dado a través del tiempo, es así que solo dos horas de precipitación continua, las crecidas podrían arrasar lo que el hombre ha tardado en construir en años.

El agua en ingeniería es un problema, tanto por escases o abundancia, de manera que las lluvias pueden tener efectos beneficiosos y perjudiciales. Los ríos por lo general tienen a crecer significativamente gracias a las lluvias, esto dependerá del tiempo e intensidad de esta, por lo que esto variará dependiendo de la época de precipitaciones. En la zona de estudio la época húmeda se da en los meses de diciembre a mayo, debido a la presencia en las costas de la corriente cálida de El Niño.

La variabilidad climática de la costa, cuando las temperaturas aumentan considerablemente, como en el año 1983, se refleja en las precipitaciones que alcanzaron aproximadamente $2823 \mathrm{~mm}$, en el año 1997 con precipitaciones de hasta $2007 \mathrm{~mm}$ [8].

El río Manglaralto como corriente natural fluye de manera ocasional, en los meses húmedos, mientras que, en los meses considerados secos, se presenta la denominada garua, la cual es una precipitación muy baja, manteniendo húmedo el sistema hídrico de la cuenca, destacando la ocurrencia de flujo superficial en la cuenca alta.

La ausencia de mapas de inundación genera un problema a los habitantes de la cuenca del río Manglaralto, dado que al aumentar la población surge la necesidad de espacios habitables para vivienda, lo que lleva a urbanizar las riberas del río y con ello aumentar riesgo a ser alcanzado por inundaciones.

Los riesgos de ocurrencia de inundaciones, principalmente en eventos conocidos como fenómeno del 
Niño, en la que las precipitaciones aumentan considerablemente por encima de la media anual, dado que no son eventos aislados sino que se repiten periódicamente en el tiempo, lo que hace posible que se repita en los próximos años causando daños y desastres considerables a las estructuras físicas emplazadas en las riberas, siendo necesario un mayor conocimiento previo para mitigar aquello advertir las zonas vulnerables a inundación.

"Las características del territorio inciden en la intensidad y el comportamiento de los fenómenos naturales" [9], entonces los mapas de zonas de inundación dependen de las características físicas del medio, en el caso de la cuenca del río Manglaralto, las características topográficas están marcadas por dos situaciones, la primera la cuenca alta presenta pendientes por encima del 30\% típico de montaña, mientras que la cuenca media y baja presenta un valle que es donde se podría generar problemas de inundación, el tirante del cauce del río varía espacialmente, siendo en la cuenca baja donde se presenta la mayor magnitud de este parámetro, el agua fluye hacia el mar. Con el aumento de la intensidad de lluvia, la zona de evacuación del agua superficial se desacelera, llegando a represarse en la parte costera lo que provoca desborde lateral en sus riberas, volviendo más profundo sus aguas arriba, por otro lado el cauce del río Manglaralto en la cuenca media y alta es estrecho donde sus riberas son tratadas como tierra agrícola en la cuenca media y espacios turísticos en la cuenca alta debido a que la mayor parte del tiempo presenta un caudal mínimo y un ambiente tropical durante las diferentes estaciones del año.

En el presente trabajo la modelación hidrológica se fundamenta en el estudio de escenarios, estos son la situación natural, actual y futura los cuales presentan cambios en el uso del suelo con el paso del tiempo, así como el caudal.

Para la elaboración de escenarios fue necesario el uso de los programas HEC HMS en el que se generan los hidrogramas de crecida [10] y HEC RAS que simula la lámina de agua que discurre en el río Manglaralto determinando las zonas inundables [11] .

El objetivo principal del proyecto es obtener mapas de inundación del río Manglaralto para diferentes escenarios de uso de suelo y ocurrencia de lluvias intensas, con lo cual se pretende mejorar el conocimiento sobre la incidencia del asentamiento poblacional en las proximidades del río y el aumento del riesgo por inundación.

\section{METODOLOGÍA}

La metodología empleada para la investigación se desarrolla en tres fases. En la a Fig. 1 se observa el resumen de la metodología implementada.

La Fase 1, corresponde a la investigación bibliográfica acerca de la temática en hidrología e hidráulica, así como la revisión y análisis de estudios anteriores de tesis doctorales, de grados y maestría, también de proyectos relacionados a recursos hídricos y en especial a la cuenca Manglaralto entre estos tenemos:

Estudio Hidrogeológico de la cuenca baja del río Manglaralto [12].

Las Lecciones del Niño, Memorias del Fenómeno del Niño 1997-1998 Retos y Propuestas para la Región Andina [8]

Análisis hidrogeológico de la cuenca del río Manglaralto para la caracterización de sus sistemas acuíferos [7].

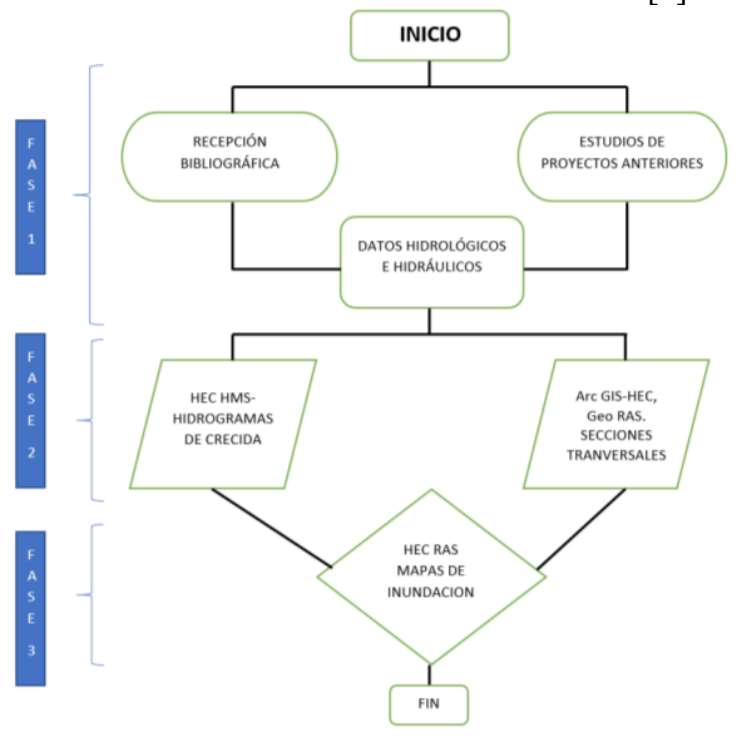

Fig. 1 Diagrama de flujo de la metodología

La Fase 2, está relacionada con la obtención de los datos y es donde se desarrollan los hidrogramas de crecida para tormentas de 24 horas por medio del software HEC HMS.

La cuenca del río Manglaralto fue dividida en 19 subcuencas que alimentan y forman parte del cauce principal como se muestra en la Fig. 2. El tramo de estudio es de ABC del cauce principal de la cuenca.

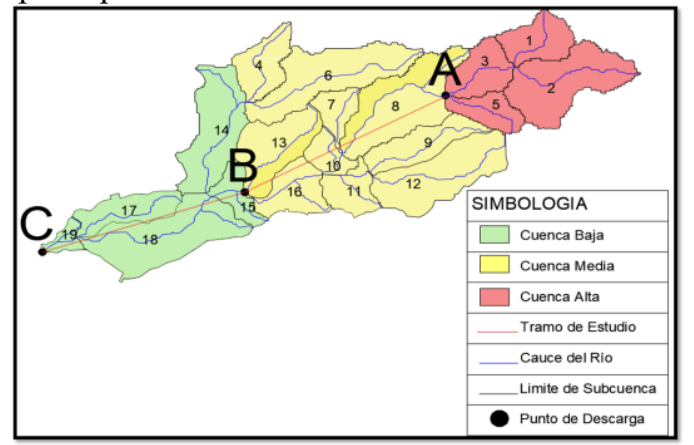

Fig. 2 Subcuencas y tramo de estudio

Los hidrogramas de crecida se obtuvieron en base a resultados de cálculo de parámetros según las características físicas de cada subcuenca y el hietograma de crecida para los diferentes escenarios a través del Método de Bloque Interno IDF (Intensidad Duración y Frecuencia) basado en periodos 
de retorno diferentes según el escenario basados en la siguiente expresión:

$$
i=\frac{c}{t^{f}+e}
$$

$i=$ intensidad

$t=$ tiempo de concentración

donde los valores de c, e y f se muestran en la tabla I.

TABLA I

Valores de c, e y $\mathrm{f}$

\begin{tabular}{|c|c|c|c|}
\hline \multirow{2}{*}{$\begin{array}{c}\text { Recurrencia } \\
\text { (Años) }\end{array}$} & \multicolumn{3}{|c|}{ Factores } \\
\cline { 2 - 4 } c & e & f \\
\hline 5 & 570.75 & 2.35 & 0.5 \\
\hline 10 & 521 & 1.49 & 0.45 \\
\hline 25 & 486.47 & 0.88 & 0.4 \\
\hline 50 & 471.72 & 0.59 & 0.37 \\
\hline 100 & 463.15 & 0.38 & 0.35 \\
\hline
\end{tabular}

En la Fase 3, se realiza la integración de toda la información obtenida e importada al programa HEC RAS, para la elaboración de los mapas de inundación.

\section{RESULTADOS}

\section{A. Datos Hidrológicos e Hidráulicos}

1) Hietogramas

Los escenarios 1 y 2 corresponden a la situación natural y actual respectivamente y se efectuó con el hietograma con recurrencia de 5 años Fig. 3 y el escenario 3 con recurrencia de 50 años como se observa en la Fig. 4.

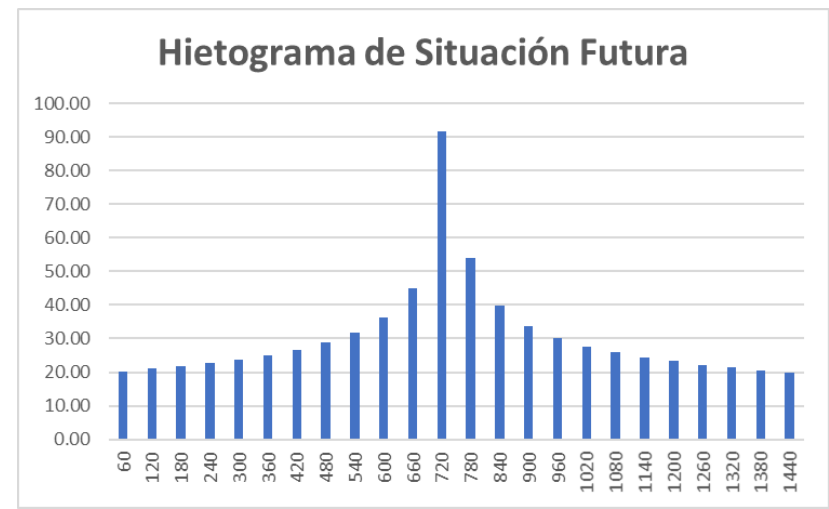

Fig. 3 Hietograma de precipitación (mm) vs tiempo (minutos)

2) Número de Curva (NC)

Para el cálculo de número de curva fue necesario el umbral de escorrentía $\left(\mathrm{P}_{\mathrm{o}}\right)$ para cada subcuenca según el grupo de suelo: A, B, C, y D (Fig.5).
$\mathrm{NC}=$ Número de Curva

$$
N C=\frac{25400}{254+P_{o} / 0,2}
$$

$\mathrm{Po}=$ Umbral de escorrentía

La característica hidrológica donde denota cultivo según las curvas de nivel y $\mathrm{R}$ denota cultivo según la línea de máxima pendiente en donde las pendientes son evaluadas en $<3 \%$ o $>3 \%$, debido a que a medida que aumenta la urbanización en base al cálculo de la situación actual del terreno, por ello se determinó $-5 \%$ para la situación natural y $+5 \%$ para la situación futura como se muestra en la tabla II.

TABLA II

NÚMERO DE CURVA PARA SUBCUENCA

\begin{tabular}{|c|c|c|c|c|c|}
\hline \multirow{2}{*}{$\begin{array}{c}\text { Sub } \\
\text { cuenca }\end{array}$} & Tipo de Suelo & Po & $\begin{array}{c}\text { Situación } \\
\text { Natural }\end{array}$ & $\begin{array}{c}\text { Situación } \\
\text { Actual }\end{array}$ & $\begin{array}{c}\text { Situación } \\
\text { Futura }\end{array}$ \\
\cline { 3 - 6 } & & $\mathbf{- 5 \%}$ & $\mathbf{N C}$ & $\mathbf{+ 5 \%}$ \\
\hline 1 & área protegida & 10 & 79 & 84 & 88 \\
\hline 2 & área protegida & 10 & 79 & 84 & 88 \\
\hline 3 & área protegida & 10 & 79 & 84 & 88 \\
\hline 4 & B & 42 & 52 & 55 & 57 \\
\hline 5 & área protegida & 16 & 72 & 76 & 80 \\
\hline 6 & B & 42 & 52 & 55 & 57 \\
\hline 7 & B & 34 & 57 & 60 & 63 \\
\hline 8 & C & 22 & 66 & 70 & 73 \\
\hline 9 & C & 17 & 71 & 75 & 79 \\
\hline 10 & D & 10 & 79 & 84 & 88 \\
\hline 11 & D & 17 & 71 & 75 & 79 \\
\hline 12 & B & 17 & 71 & 75 & 79 \\
\hline 13 & D & 10 & 79 & 84 & 88 \\
\hline 14 & C & 10 & 79 & 84 & 88 \\
\hline 15 & D & 8 & 82 & 86 & 91 \\
\hline 16 & D & 10 & 79 & 84 & 88 \\
\hline 17 & D & 4 & 88 & 93 & 97 \\
\hline 18 & D & 6 & 85 & 89 & 94 \\
\hline 19 & D & 8 & 82 & 86 & 91 \\
\hline
\end{tabular}

3) Tiempo de Retardo

Cada subcuenca cuenta con propias características por ende la diferencia del tiempo que tarda una gota en salir de ella dependerá del tiempo de concentración de cada subcuenca en donde interviene la longitud del cauce y la pendiente de cada una en base a la siguiente expresión:

$$
t_{c}=K L^{0.770} S^{-0.385}
$$

$\mathrm{K}=$ Coeficiente de conversión de unidades, en donde es 0.0195 para S.I.

$\mathrm{L}=$ Longitud del cauce

$\mathrm{S}=$ Pendiente promedio del cauce principal

$$
T R=60 \% T c
$$

Donde el Tc es el tiempo de concentración y TR es el tiempo de retorno (Tabla III). 
TABLA III

VALORES DE TIEMPO DE CONCENTRACIÓN Y RETORNO

\begin{tabular}{|c|c|c|}
\hline SUBCUENCA & TC & TR \\
\hline 1 & 14.71 & 8.83 \\
\hline 2 & 29.61 & 17.77 \\
\hline 3 & 19.66 & 11.80 \\
\hline 4 & 32.37 & 19.42 \\
\hline 5 & 19.33 & 11.60 \\
\hline 6 & 67.35 & 40.41 \\
\hline 7 & 23.43 & 14.06 \\
\hline 8 & 45.62 & 27.37 \\
\hline 9 & 43.74 & 26.24 \\
\hline 10 & 25.94 & 15.56 \\
\hline 11 & 24.45 & 14.67 \\
\hline 12 & 51.89 & 31.13 \\
\hline 13 & 45.56 & 27.34 \\
\hline 14 & 66.06 & 39.64 \\
\hline 15 & 35.33 & 21.20 \\
\hline 16 & 33.06 & 19.83 \\
\hline 17 & 48.76 & 29.26 \\
\hline 18 & 152.64 & 91.58 \\
\hline 19 & 78.77 & 47.26 \\
\hline
\end{tabular}

\section{B. Hidrogramas de Crecida}

Los hidrogramas de crecidas fueron realizados para los tramos de estudio ( $\mathrm{AB}$ y $\mathrm{BC})$, considerando la escorrentía superficial de cada subcuenca que convergen en una zona en común para los tres escenarios según el uso de suelo de la zona de estudio tanto para la situación natural, actual y futura.

\section{1) Situación Natural}

Tramo AB

Los datos calculados indican que el caudal máximo acumulado se da a las 13 horas del día con una magnitud de $270.4 \mathrm{~m}^{3} / \mathrm{s}$, el cual es producto de lo que aporta la subcuenca 13 y 16 directamente más lo que se conduce aguas arriba. El hidrograma del cauce del tramo BC indica que el caudal es exclusivo del evento de precipitación (Fig. 4)

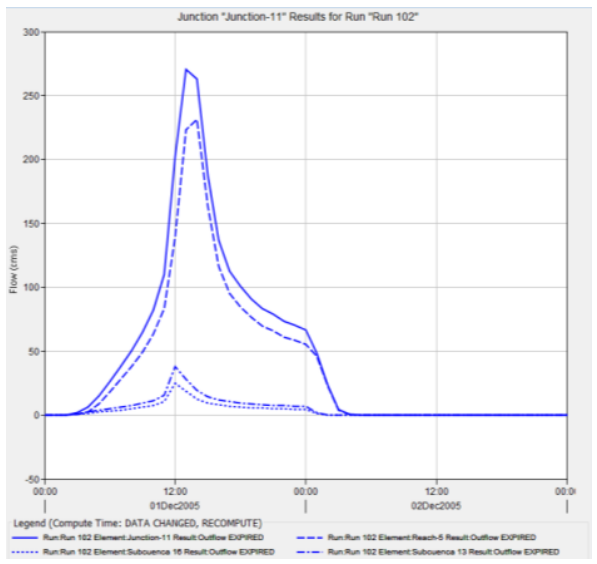

Fig. 4 Hidrograma de crecida Escenario 1

Tramo BC

Los datos calculados indican que el caudal máximo se da a las 16 horas del día con una magnitud de $366 \mathrm{~m}^{3} / \mathrm{s}$, el cual es producto de lo que aporta directamente la subcuenca 19 y lo conduce el tramo 9 de las subcuencas aguas arriba (Fig. 5)

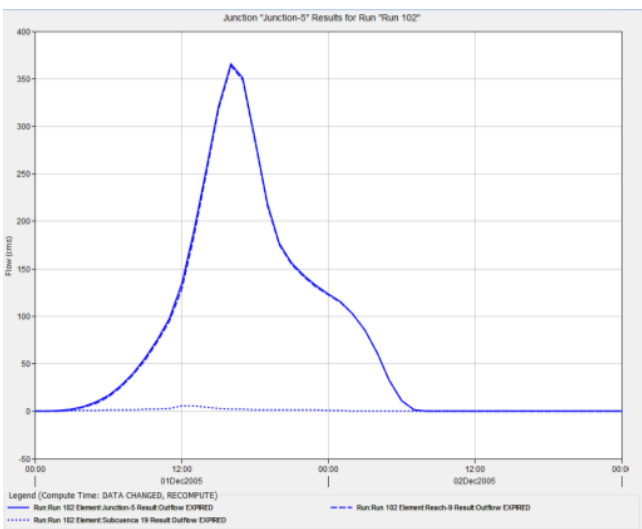

Fig. 5 Hidrograma de crecida Escenario 1

\section{2) Situación Actual}

\section{Tramo AB}

Los datos de salida en el extremo del tramo que se han calculados indican que el caudal máximo acumulado se da a las 13 horas del día con una magnitud de $319.7 \mathrm{~m}^{3} / \mathrm{s}$, el cual es producto de lo que aporta la subcuenca 13 y 16 directamente más lo que conduce los tramos aguas arriba (Fig.6).

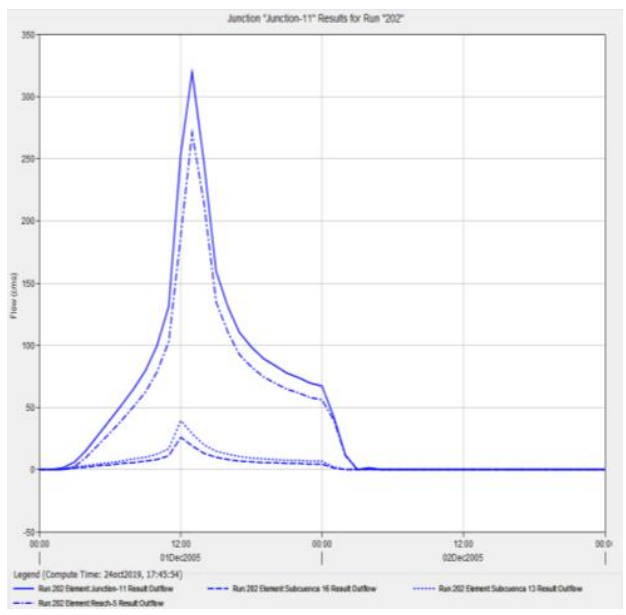

Fig. 6 Hidrograma de crecida Escenario 2

\section{Tramo BC}

Los datos calculados indican que el caudal máximo se da a las 16 horas del día con una magnitud de $405.7 \mathrm{~m}^{3} / \mathrm{s}$, el cual es producto de lo que aporta directamente la subcuenca $19 \mathrm{y}$ lo conduce el tramo 9 de las subcuencas aguas arriba (Fig.7). 


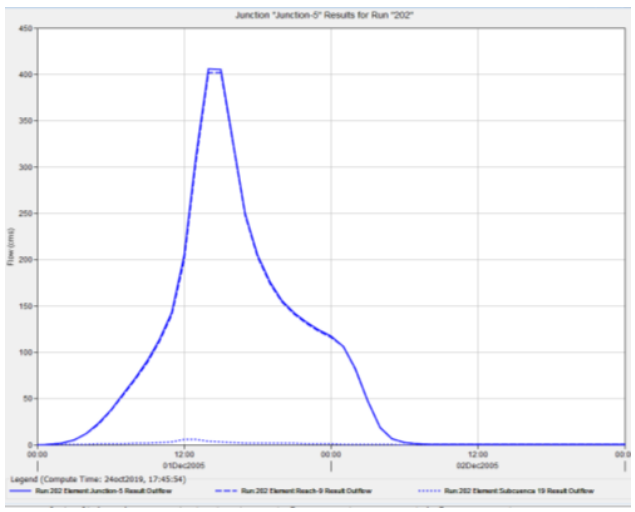

Fig. 7 Hidrograma de crecida Escenario 2

\section{3) Situación Futura}

Tramo AB

Los datos de salida en el extremo del tramo que se han calculados indican que el caudal máximo acumulado se da a las 13 horas del día con una magnitud de $605.2 \mathrm{~m}^{3} / \mathrm{s}$, el cual es producto de lo que aporta la subcuenca 13 y 16 directamente más lo que conduce los tramos aguas arriba (Fig. 8).

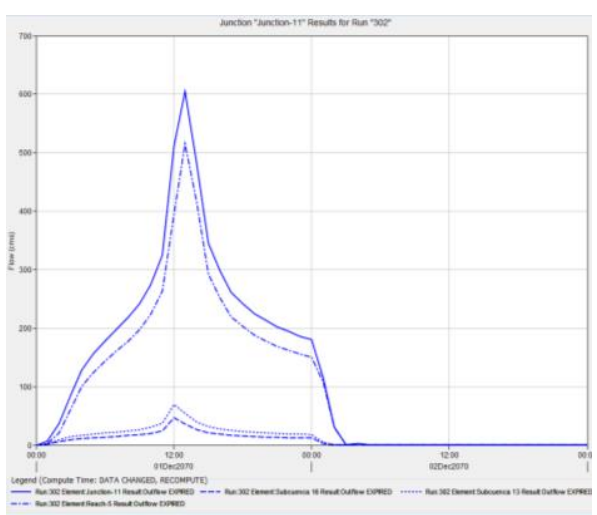

Fig. 8 Hidrograma de crecida Escenario 3

\section{Tramo BC}

Los datos calculados indican que el caudal máximo se da a las 16 horas del día con una magnitud de $824.9 \mathrm{~m}^{3} / \mathrm{s}$, el cual es producto de lo que aporta directamente la subcuenca 19 y lo conduce el tramo 9 de las subcuencas aguas arriba (Fig.9).

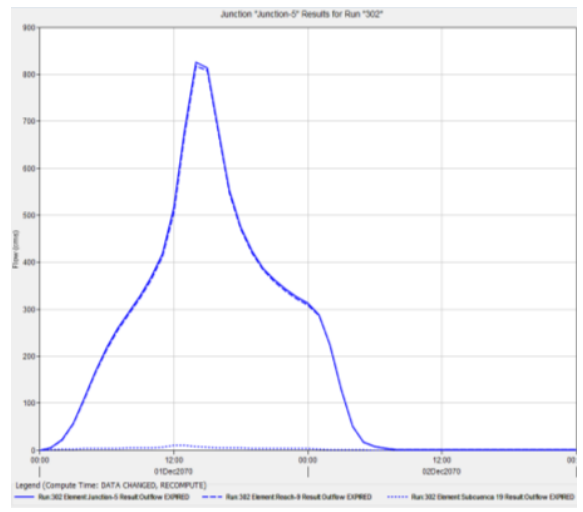

Fig. 9 Hidrograma de crecida Escenario 3

\section{Secciones Transversales}

A la vez en esta fase se digitaliza la geometría Fig. 10 con el software ArcGIS para las secciones transversales Fig. 11 graficadas cada 75 metros y transportadas a HEC-RAS bajo la extensión HEC GeoRAS.
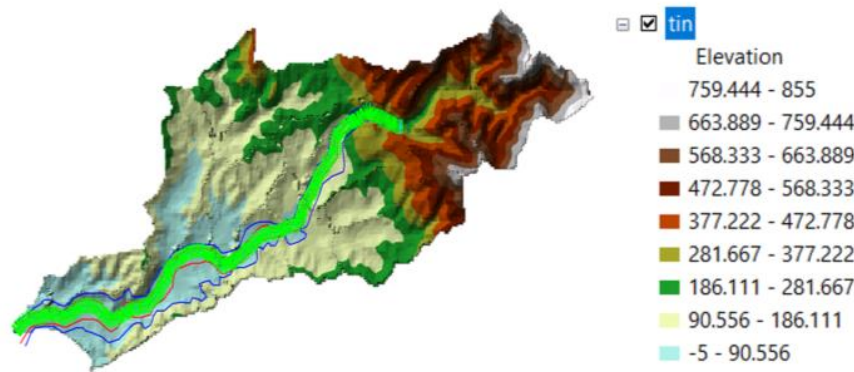

Fig. 10 Generación de geografía con HEC GeoRAS

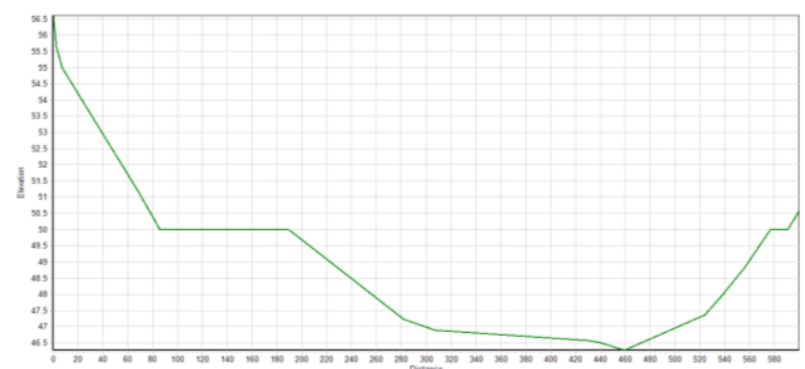

Fig. 11 Sección Transversal

\section{Mapas de Inundación}

Para el ingreso de resultados y modelación del agua que discurre en la cuenca Manglaralto se subdividió en 2 tramos principales.

Para la determinación de zonas inundables en los diferentes escenarios fueron necesarios criterios que determinan los mapas de inundación y nivel de amenaza para los tres escenarios en los que se representan las posibles amenazas hídricas respecto al flujo en la cuenca.

Una vez conocida el área de inundación obtenida por el programa HEC RAS, los criterios para determinar los mapas 
de amenaza por inundación en la cuenca, indican un rango que va desde una amenaza residual a amenaza media.

Los resultados obtenidos de la modelación hidrodinámica de un evento de crecida para periodos de retorno de 5 y 50 años, se establecen bajo las siguientes condiciones, considerando los siguientes parámetros de contorno:

Geometría

- Longitud del tramo: $13.875 \mathrm{~km}$

- Número de secciones transversales: 187

- Longitud de las secciones transversales:100

- Distancia entre las secciones transversales:75

Condiciones de frontera

- Flujo no permanente (hidrogramas)

- Pendiente del tramo: 0.019

- Coeficiente de Manning:

Margen Izquierdo y Derecho: 0.0280 .028

Centro:0.03

Variación de los coeficientes de contracción en estructuras de paso para escenarios 2 y 3 .

Contracción 0.6

Expansión 0.8

\section{1) Simulación Escenario 1}

Los resultados de la simulación del Escenario 1, que corresponde a la situación natural, indican que el cauce fluye con normalidad, notando donde se presenta la llanura de inundación en el espacio, consiguiendo como resultado que esta se da en los sectores que poseen menor pendiente. En la Fig. 12 se representa el perfil longitud del río. En la Fig. 13 se presenta el mapa de inundación en el que se observan los desbordes superficiales del río.

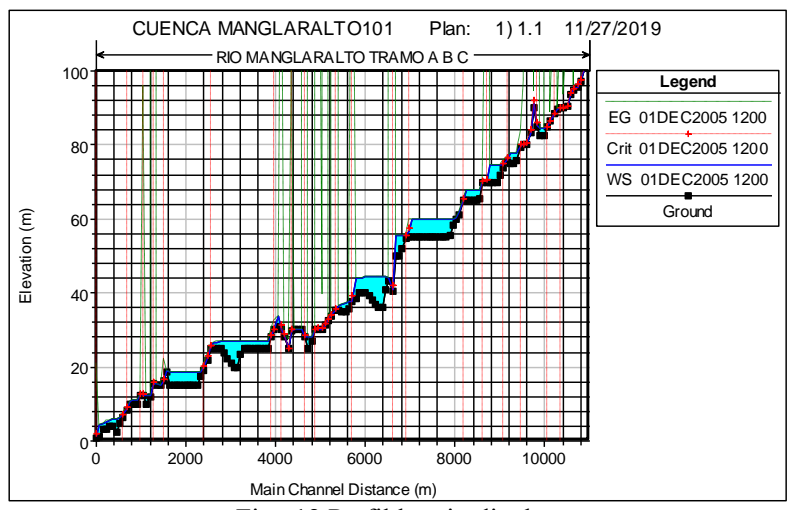

Fig. 12 Perfil longitudinal

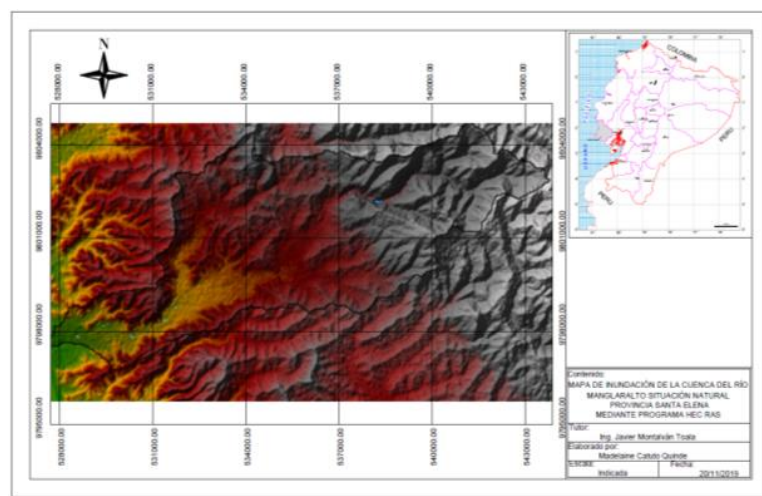

Fig. 13 Mapa de inundación - Situacion natural del terreno

2) Simulación Escenario 2

Los resultados de la simulación del Escenario 2, que corresponde a la situación actual, indican que el cauce fluye con normalidad, notando donde se presenta la llanura de inundación en el espacio, consiguiendo como resultado que esta se da en los sectores que poseen menor pendiente.

La simulación muestra en la sección transversal "XS 1050" Fig. 14, es una de las secciones donde existe un puente actualmente y el mapa generado en la Fig. 15.

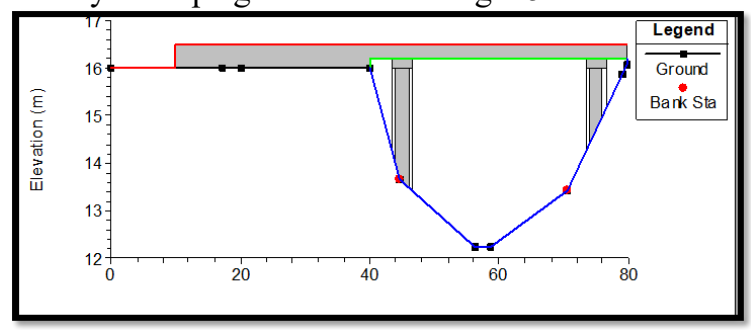

Fig. 14 Estructura de paso existente puente E-15

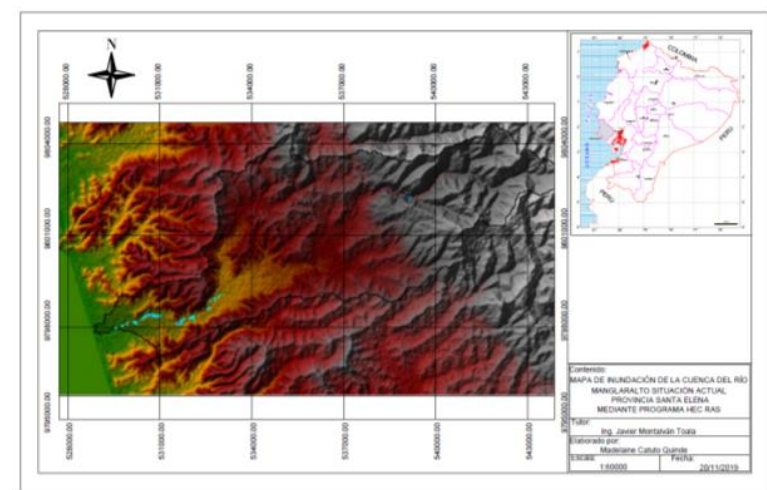

Fig. 15 Mapa de inundación - Situacion actual del terreno

3) Simulación Escenario 3

Los resultados de la simulación del Escenario 3, que corresponde a la situación futura, indican que el cauce fluye con normalidad, notando donde se presenta la llanura de inundación en el espacio, consiguiendo como resultado que esta se da en los sectores que poseen menor pendiente. En la Fig. 16 se representa los resultados de la llanura de inundación 
en donde se observa acumulación de flujos de agua más notables que los dos escenarios anteriores.

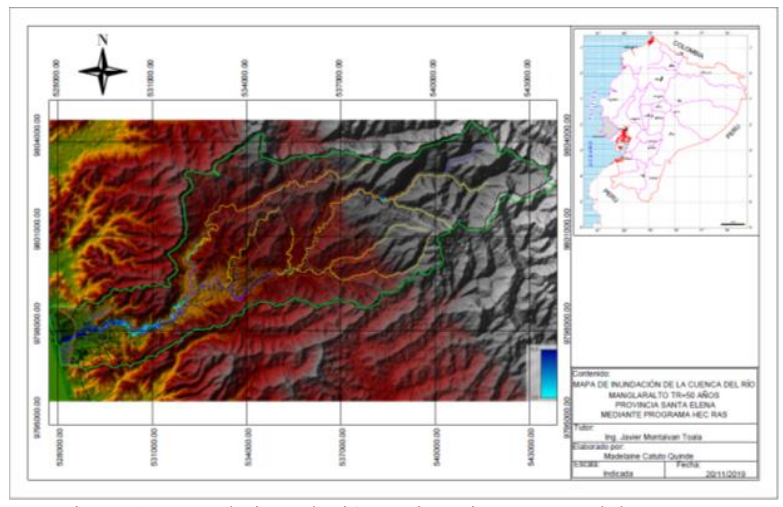

Fig. 16 Mapa de inundación - Situacion Futura del terreno

\section{DISCUSIÓN}

La cuenca del río Manglaralto posee un caudal en el tramo BC para periodos de retorno de 5 y 50 años de 405.7 $\mathrm{m}^{3} / \mathrm{s}$ y $824.9 \mathrm{~m}^{3} / \mathrm{s}$ respectivamente, lo que podría ocasionar áreas de inundación en este tramo. Las precipitaciones en la cuenca entre los años 1962 y 2014 varían entre $200-700$ mm anuales, considerando que las máximas precipitaciones se dan en los eventos conocidos como el fenómeno del Niño [13]. En este trabajo se ha considerado un evento de precipitaciones durante 24 horas por medio de una simulación hidrológica, en la que la máxima precipitación para periodo de retorno de $5 \mathrm{y}$ 50 años fue de $56.53 \mathrm{~mm}$ y $91.79 \mathrm{~mm}$ en ese orden, entonces los resultados obtenidos en la simulación son excesivos comparados con los registros de precipitaciones mencionados por [13].

En esta investigación se propone tres escenarios sin actividad antrópica, y cuando el hombre realiza actividades sobre la cuenca, siempre considerando el modelo hidrológico de 24 horas de precipitación, consiguiendo para cada uno de estos escenarios, áreas propensas a inundarse. En el trabajo de [14] realizan un mapa de susceptibilidad a inundaciones en el norte de Irán, el cual considera básicamente la topografía y usos de suelo para establecer estas zonas, mientras que el presente estudio considera también la intensidad de las precipitaciones. Estas precipitaciones calculadas pueden ser por un tiempo prolongado y su escurrimiento superficial es el mismo debido a que los factores climáticos como la evaporación generan perdida mínima del flujo.

El clima en la cuenca de estudio es semi-árido, lo que hace que ocurran problemas de inundación principalmente en los años más húmedos en el escenario natural. Cuando el hombre está presente a pesar de ser un clima seco el riesgo de zonas de inundación aumenta debido a las actividades de estructuras antrópicas lo que hace disminuir el cauce del río y aumente el tirante y con ello que las áreas de las riberas principalmente en la cuenca baja se produzcan inundaciones. En la cuenca en
Poyang County en China las precipitaciones media anual alcanza los $1600 \mathrm{~mm}$ es decir una zona húmeda, en la que las edificaciones están construidas muy próximas a las riberas del río, ocasionando inundación en las zonas habitables [15].

\section{CONCLUSIONES}

- En el presente trabajo se elaboraron tres mapas de inundación, de los que el escenario 1 que corresponde a la situación natural de la cuenca, es decir sin presencia de actividades humanas, se concluye que bajo esas condiciones no existe riesgo de inundaciones a lo largo del tramo $\mathrm{ABC}$.

- $\quad$ En el escenario 2 (situación actual) y en el escenario 3 (situación futura), se realiza la simulación con un periodo de retorno de 10 años y 50 años respectivamente, así como los diques y estructuras de paso se encuentran representados mediante la modificación de los parámetros de expansión y contracción, para el escenario 2, se presentan inundaciones aisladas en varios sectores de la cuenca media, existiendo un bajo riesgo de inundación, mientras que en el escenario 3, existe un potencial riesgo de inundación en la cuenca baja.

- La simulación numérica del flujo superficial que discurre por el río Manglaralto, se concluye que la escorrentía depende de los factores físicos propios de la cuenca, dependiendo principalmente de las precipitaciones, así como de la topografía que incide directamente en las pendientes existentes, dado que en el tramo $\mathrm{AB}$ la pendiente es de 5\%, por lo que no existe riesgo de inundaciones en ambos escenarios, mientras que en el tramo $\mathrm{BC}$ la pendiente es de $1 \%$ y la afluencia de cauces hacía el río principal, lleva a que el riesgo aumente en aquella zona junto con el aumento de precipitaciones.

- $\quad$ Se estima que las poblaciones afectadas por el escenario 2 y escenario 3 de los tramos $\mathrm{AB}$ y $\mathrm{BC}$ de las comunidades Manglaralto $\mathrm{y}$ Dos mangas en donde ocurre un desbordamiento lateral del río a 50 metros del eje principal donde las poblaciones afectadas para el caso 1 es Dos Mangas, mientras que en el caso 2 las hacienda La Ponderosa, San Luis, La Palma, Dos Mangas se ven afectados por el encuentro de los alimentadores del río y al aumento de caudal debido y exclusivamente a las precipitaciones.

- Los mapas obtenidos de la simulación concluyen que los escenarios donde se presentan las mayores precipitaciones, considerando las estructuras de paso y diques para aumentar la recarga del acuífero que son representados por los parámetros de contracción y expansión, permiten realizar precauciones respecto a alertas tempranas de evacuación de personas y animales en riesgo de inundación, así como el correcto uso del suelo, donde no se podrá realizar obras civiles de vivienda. 
Los autores agradecen a la Junta Administradora de Agua Potable de Manglaralto (JAAPMAN) por su apoyo logístico en el desarrollo de esta investigación, así como al CIPATESPOL en la obtención y procesamiento de datos y a la UPSE por medio del proyecto No. 91870000.0000 .382444 quien financió esta investigación.

\section{REFERENCIAS}

[1] Burbano, N. Becerra, S. Pasquel, E. (2011). Introducción a la hidrogeología del Ecuador. Instituto Nacional de Hidrología y Meteorología. Quito, Ecuador.

[2] Morante, F.; Montalván. F.; Carrión, P.; Herrera, G.; Elorza, F.; Pilco. D; Solorzano, J. (2019). Hydrochemical And Geological Correlation To Establish The Salinity Of Groundwater Of The Coastal Aquifer Of The Manglaralto Ecuador River Basin.

[3] CIPAT-ESPOL (Guayaquil, Ecuador), SENAGUA (2014). Proyecto mapa Hidrogeológico escala 1:250.000. Informe inédito.

[4] Arévalo, M. (2017). Propuesta de diseño de construcción, acondicionamiento de un pozo de agua y su incidencia en la vulnerabilidad del acuífero costero en Manglaralto. Undergraduate, Escuela Superior Politécnica Del Litoral, Guayaquil.

[5] Campoverde, J.; Fajardo, I. (2018). Contribución de la geología aplicada para la mejora en el diseño de un dique en la subcuenca del río Manglaralto, provincia de Santa Elena. Undergraduate, Escuela Superior Politécnica Del Litoral, Guayaquil.

[6] Herrera, G. (2015). Estudio para un Modelo de Gestión de un Acuífero Costero, mediante Metodologías Participativas y Análisis Geoestadístico en el marco del Desarrollo Local. Manglaralto, Ecuador. Doctoral, Polytechnic University of Madrid.

[7] Valencia, J. I. (2017). Análisis hidrogeológico de la cuenca del río Manglaralto para la caracterización de sus sistemas acuíferos. Undergraduate, Escuela Superior Politécnica Del Litoral, Guayaquil.

[8] Corporación Andina de Fomento (2000). Las lecciones de El Niño. Vol. 4: Ecuador. Memorias.

[9] Espinoza, G.A. y E.R. Hakey (1989). Riesgos Climáticos: Evidencias en Chile Central En: Ecología del Paisaje en Chile Central, estudio sobre sus espacios montañosos. Pp41-52 Fuentes y Prenafeta (eds). Edic. P. Universidad Católica de Chile.

[10] US ACE (US Army Corps of Engineers), 2008. Hydrologic modeling system (HEC-HMS) application guide: Version 3.1.0. Davis, CA: Institute for Water Resources, Hydrologic Engineering Center.

[11] US Army Corps of Engineers (USACE), HEC-RAS (1998) River Analysis System, User "s Manual Version 2.2, Hydrologic Engineering Center, Davis, California.

[12] Amado, J. (1990). "Estudio Hidrogeológico de la cuenca Baja del río Manglaralto, Provincia del Guayas" Guayas-Ecuador. ESPOL.

[13] Gricelda Herrera Franco., Paúl Carrión Mero and Josué Briones Bitar (2019). Prácticas de gestión para una comunidad sostenible y su incidencia en el desarrollo, Manglaralto-Santa Elena, Ecuador.

[14] Alireza Arabameri, Khalil Rezaei, Artemi Cerdà, Christian Conoscenti, Zahra Kalantari (2019). A comparison of statistical methods and multicriteria decision making to map flood hazard susceptibility in Northern Iran. Science of the Total Environment 660 (2019) 443-458.

[15] Haoyuan Hong, Paraskevas Tsangaratos, Ioanna Ilia, Junzhi Liua, AXing Zhua, Wei Chen (2018). Application of fuzzy weight of evidence and data mining techniques in construction of flood susceptibility map of Poyang County, China. Science of the Total Environment 625 (2018) $575-588$.

$1^{\text {th }}$ LACCEI International Multi-Conference for Engineering, Education, and Technology: "Engineering, Integration, and Alliances for a Sustainable Development" "Hemispheric Cooperation for Competitiveness and Prosperity on a Knowledge-Based Economy", 29-31 July 2020, Buenos Aires, Argentina. 